\title{
Abiraterone Acetate
}

\section{Abstract}

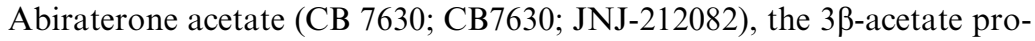
drug of abiraterone, is structurally related to ketoconazole and is being developed by Cougar Biotechnology as a hormonal therapy for advanced prostate and breast cancers. As a selective inhibitor of adrenal androgens, it is thought to be a safer product than existing second-line hormonal therapies. This review discusses the key development milestones and therapeutic trials of this drug.
\end{abstract}

\section{Introduction}

Abiraterone acetate is structurally related to ketoconazole and is the $3 \beta$-acetate prodrug of abiraterone. Abiraterone acetate is being developed by Cougar Biotechnology (a subsidiary of Johnson \& Johnson) as a hormonal therapy for advanced prostate and breast cancers. This orally active agent was first synthesized at the Institute of Cancer Research (ICR) in the UK as part of a BTG-funded program, and subsequently licensed to Cougar Biotechnology. It irreversibly inhibits the steroidal enzyme $17 \alpha$-hydroxylase/ $\mathrm{C}_{17-20}$ lyase (also known as cytochrome $\mathrm{P} 450$ [CYP]17A1 or P450c17), a CYP complex that is involved in testosterone production. Phase III trials in men with castration-resistant prostate cancer (CRPC) and a phase I/II trial in advanced breast cancer are underway.

Abiraterone acetate is believed to have potential advantages over existing second-line hormonal therapies in patients with prostate cancer because it selectively inhibits adrenal androgens, making the product safer to use. Cougar Biotechnology has stated that the drug has a potential role as a second-line hormonal therapy for patients with advanced prostate cancer resistant to first-line hormonal treatment or first-line docetaxel-based chemotherapy. ${ }^{[1]}$

In July 2009, Cougar Biotechnology became a wholly owned subsidiary of Johnson \& Johnson. ${ }^{[2]}$

\subsection{Company Agreements}

If approved, abiraterone acetate will be commercialized and marketed by Ortho Biotech in the US and by Janssen Pharmaceuticals in other countries worldwide. ${ }^{[3]}$

Cougar Biotechnology itself licensed the product from BTG plc. in April 2004. This agreement granted Cougar Biotechnology exclusive worldwide rights to develop and commercialize abiraterone acetate, while BTG plc. received an upfront cash payment and is entitled to milestones payments and royalties on sales. ${ }^{[4]}$

Boehringer Ingelheim had an exclusive option to license the development and marketing rights to abiraterone acetate. However, the company chose not to exercise the option.

This drug profile has been extracted from Wolters Kluwer's Adis ${ }^{\mathrm{TM}} R \& D$ Insight drug pipeline database. $R \& D$ Insight tracks and evaluates drug development worldwide through the entire development process, from discovery, through pre-clinical and clinical studies to market launch. This is an open access article published under the terms of the Creative Commons License "Attribution-NonCommercial-NoDerivative 3.0" (http://creative commons.org/licenses/by-nc-nd/3.0/) which permits non-commercial use, distribution, and reproduction, provided the original work is properly cited and not altered. 


\subsection{Key Development Milestones}

\subsubsection{Prostate Cancer}

Cougar Biotechnology expects to file marketing applications for abiraterone acetate in the US and Europe before the end of 2010, based on interim data from the phase III COU-AA-301 trial (NCT00638690). The randomized, placebocontrolled trial is investigating abiraterone acetate in combination with low-dose prednisone or prednisolone in 1195 patients with metastatic CRPC that has progressed following treatment with one or two chemotherapy regimens, at least one of which was docetaxel. ${ }^{[5]}$ In September 2010 , this trial was unblinded after a recommendation from the Independent Data Monitoring Committee, after a prespecified interim analysis showed a statistically significant improvement in overall survival and an acceptable safety profile. It was also recommended that patients receiving placebo be offered abiraterone acetate. Cougar Biotechnology planned to initiate an early access program in the US in October 2010, with expansion to other sites in the months following. ${ }^{[3,6,7]}$

Another phase III trial is underway, evaluating abiraterone acetate plus prednisone or placebo plus prednisone in patients with metastatic CRPC from North America, Europe, and Australia (NCT00887198). The primary endpoints are progression-free survival and overall survival. ${ }^{[5,8,9]}$ Patient enrollment was completed in May 2010.

A phase I/II trial examined daily oral abiraterone acetate as a second-line therapy in chemotherapynaive CRPC progressing despite treatment with luteinizing hormone-releasing hormone (LHRH) analogs and multiple other hormonal therapies (NCT00473746). The study was conducted at the University of California, San Francisco (UCSF), USA, Comprehensive Cancer Center, with preliminary results presented..$^{[1,10,11]}$ Johnson \& Johnson, Cougar Biotechnology, and UCSF are also conducting a phase II clinical trial to evaluate the efficacy of abiraterone acetate in approximately 46 patients with CRPC who have experienced disease progression on ketoconazole (NCT01199146).

In November 2009, Cougar Biotechnology initiated a phase II study of abiraterone acetate as a neo-adjuvant, in combination with prednisone and a LHRH analog, in approximately 66 patients with prostate cancer (NCT01088529).

Cougar Biotechnology and the M.D. Anderson Cancer Center are conducting an observational study of continuous oral dosing of abiraterone acetate in 60 patients with CRPC, evaluating the effect of the drug on levels of testosterone and DHT in bone marrow plasma (NCT00544440).

Preliminary results were reported from a phase II study evaluating the anti-tumor efficacy and safety of abiraterone acetate in combination with prednisone in 58 patients with advanced prostate cancer who had failed androgen deprivation and docetaxel-based chemotherapy in the US and UK (NCT00485303). ${ }^{[1,12]}$ Positive results were presented from a phase II trial that evaluated abiraterone acetate in 33 patients with advanced prostate cancer that had failed first-line chemotherapy with docetaxel (NCT00474383). ${ }^{[1,13]}$

In December 2006, Cougar Biotechnology initiated the phase II portion of a phase I/II study that evaluated daily dosing of abiraterone acetate as a second-line hormonal treatment for patients with chemotherapy-naive hormone-refractory prostate cancer and who have rising prostate specific antigen (PSA) levels (NCT00473512). A total of 44 patients were enrolled at trial sites in the UK. Interim data from the phase I/II study have been reported. ${ }^{[14,15]}$

In June 2010, Janssen Pharmaceutica initiated an open-label phase I trial to assess the pharmacodynamics and safety of abiraterone acetate in patients with CRPC (NCT01186484). The trial will enroll 18 patients in Japan. ${ }^{[16]}$

A phase I study to determine the effect of abiraterone acetate and prednisone on the heart is underway in the US and Canada (NCT00910754). A phase I pharmacokinetic study compared the tablet and capsule formulations of abiraterone acetate in approximately 40 patients with prostate cancer (NCT00600535).

The ICR and BTG completed three phase I trials of abiraterone acetate. The first phase I single-dose study was conducted in 16 patients with prostate cancer, and demonstrated suppression of testosterone to target levels without any serious adverse events. A second single-dose study in four patients with normal levels of testosterone showed significant suppression of testosterone le- 
vels and good tolerability. A third multiple-dose study showed complete suppression of testosterone synthesis in six patients. ${ }^{[17]}$

\subsubsection{Breast Cancer}

Cancer Research UK is enrolling approximately 74 female patients in a phase I/II, openlabel, dose-escalating study, evaluating the safety, endocrine effects, and anti-tumor activity of abiraterone acetate in estrogen or androgen receptor positive metastatic breast cancer, who have progressed on at least two lines of hormonal therapies, such as aromatase inhibitors and tamoxifen (NCT00755885). ${ }^{[18,19]}$

\subsection{Patent Information}

Abiraterone acetate is covered by two US patents (No. 5604213 and 5618 807), which expire in 2014. Patent applications have also been filed in other territories.

\section{Scientific Summary}

\section{1 Pharmacokinetics}

The tablet and capsule formulations of abiraterone were assessed in a phase I crossover study of patients with prostate cancer. Similar maximum plasma concentration $\left(\mathrm{C}_{\max }\right)$ and area under the concentration-time curve (AUC) values were obtained for both formulations and in both cases the values were higher in the fed state compared with the fasted state. $\mathrm{C}_{\max }$ values were 1469 (fed), 286 (fasted), and 1573 (fed), 324 (fasted), and AUC values were 10978 (fed), 3249 (fasted), and 10782 (fed), 3090 (fasted) for capsules and tablets, respectively. ${ }^{[20]}$

\subsection{Adverse Events}

\subsubsection{Prostate Cancer}

Phase III: Treatment with abiraterone acetate was associated with an increase in the frequency of mineralocorticoid-related adverse events, compared with placebo, in the phase III COU-AA-301 trial. The trial randomized 1195 patients with metastatic, castrate-resistant prostate cancer to treatment with either abiraterone acetate or placebo, in combination with low-dose prednisone or prednisolone. In the abiraterone and placebo groups, fluid retention occurred in $30.5 \%$ versus $22.3 \%$ of patients, respectively, and hypokalemia occurred in $17.1 \%$ versus $8.4 \%$ of patients. Grade 3/4 hypokalemia was reported in $3.8 \%$ versus $0.8 \%$ of patients and grade

Table I. Features and properties

\begin{tabular}{ll}
\hline Alternate names & CB 7630; CB7630; JNJ-212082 \\
Originator & The Institute of Cancer Research \\
Licensee(s) & Cougar Biotechnology \\
Highest development phase & III (Australia, Canada, Europe, USA) \\
Active development indications & Breast cancer, prostate cancer \\
Class & Androstenols, small molecules \\
Mechanism of action & CYP17A1 protein inhibitors \\
Chemical name & 17-(pyridin-3-yl)androsta-5,16-dien-33-yl acetate \\
Molecular formula & C26 H33 N O2 \\
CAS registry number & 154229-18-2 \\
Route of administration & PO \\
Pharmacodynamics & Inhibits androgen biosynthesis without suppressing corticosterone production in mice; suppresses \\
ATC codes & testosterone to castration levels in patients with prostate cancer \\
WHO ATC code & \\
EphMRA ATC code & L02B-B (anti-androgens) \\
Adverse events & L2B2 (cytostatic anti-androgens) \\
Occasional &
\end{tabular}


$3 / 4$ hypertension occurred in $1.3 \%$ versus $0.3 \%$ in the abiraterone acetate and placebo groups, respectively. Cardiac disorders and liver function test abnormalities were also more frequent in the abiraterone acetate group than the placebo group $(12.5 \%$ vs $9.4 \%$ and $10.4 \%$ vs $8.1 \%$, respectively). These adverse events were responsive to medical treatment. ${ }^{[3]}$

Phase II: Interim results indicated that abiraterone acetate (up to $2000 \mathrm{mg}$ /day) was well tolerated with minimal toxicity in chemotherapynaive patients with CRPC in a phase I/II study. ${ }^{[14]}$

Phase I: Abiraterone acetate was well tolerated at oral doses as high as $2000 \mathrm{mg} /$ day with minimal toxicity in 18 chemotherapy-naive patients with CRPC in a phase I study. Furthermore, no doselimiting toxicity was observed. ${ }^{[21,22]}$

Animal Toxicology: The effects of abiraterone acetate, abiraterone, and CB 7627 on WHT mice were compared with castration and the two clinically active compounds, ketoconazole and flutamide. Flutamide and castration caused significant reductions in the weight of the ventral prostate and seminal vesicles. Abiraterone acetate, abiraterone or CB 7627 once daily for 3 weeks caused significant reductions in the weights of the ventral prostate, seminal vesicles, kidneys, and testes; ketoconazole caused no reductions. ${ }^{[23]}$

\subsection{Pharmacodynamics}

\subsubsection{Cancer}

Clinical Studies: Interim results from a phase II trial in 16 evaluable patients with CRPC who had progressive disease despite treatment with LHRH analogs and multiple other therapies showed a decline in both serum and bone marrow testosterone levels to below detectable levels in all patients. Patients with depleted baseline bone marrow testosterone levels appeared to progress earlier when treated with abiraterone acetate compared with measurable baseline bone marrow testosterone levels. Further examination of bone marrow biopsies in patients treated with abiraterone acetate revealed both overexpression of androgen receptor and CYP17.[12]

Preliminary results from a phase II trial (COU-AA-002) in patients with chemotherapynaive CRPC treated with abiraterone acetate in combination with prednisone showed a decline in PSA levels of $>30 \%$ in 24 of 31 evaluable patients $(77 \%)$. A decline of $>50 \%$ was seen in $71 \%$ of patients and $26 \%$ of patients experienced a decline of PSA levels $>90 \%$. The median time to progression was not reached, and patients were continued to be treated for 10 or more months. ${ }^{[24]}$

Preliminary results from a phase II trial (COU-AA-BMA) in patients with CRPC who had progressive disease despite treatment with LHRH analogs and other therapies, showed that treatment with abiraterone acetate in combination with prednisone resulted in a decline in PSA levels of $>50 \%$ in 24 patients $(48 \%)$. Declines of $>90 \%$ were seen in six patients $(12 \%)$. Furthermore, a decline in both serum and bone marrow testosterone levels to below detectable levels was seen in all patients in the trial. ${ }^{[24]}$

In a phase I trial in three non-castrated patients with prostate cancer given abiraterone acetate $(500 \mathrm{mg} / \mathrm{day})$ for 12 days, testosterone levels were suppressed to $<2 \mathrm{nmol} / \mathrm{L}$, but remained $>0.7 \mathrm{nmol} / \mathrm{L}$. A second three-patient non-castrated cohort, treated with $800 \mathrm{mg} /$ day for 12 days, demonstrated suppression of testosterone to $<0.7 \mathrm{nmol} / \mathrm{L}$ in two patients, with a nadir of $1.7 \mathrm{nmol} / \mathrm{L}$ in the third patient. ${ }^{[25]}$

In the two single-dose clinical studies, four of six castrated patients treated with a single-dose of abiraterone acetate $500 \mathrm{mg}$ achieved suppression of testosterone to levels $<0.14 \mathrm{nmol} / \mathrm{L}$. Patients treated at the 500 and $800 \mathrm{mg}$ level experienced suppression of testosterone to castrate levels $(<2.0 \mathrm{nmol} / \mathrm{L})$. Among the patients treated with $800 \mathrm{mg} /$ day, two of three had complete suppression of testosterone down to levels comparable with those typically seen in patients treated with LHRH agonists $(<0.7 \mathrm{nmol} / \mathrm{L}){ }^{[26]}$

Preclinical Studies: Abiraterone acetate reduced plasma testosterone levels to $\leq 0.1 \mathrm{nmol} / \mathrm{L}$, despite a 3- to 4-fold increase in the plasma level of luteinizing hormone in mice. Adrenal weights were unchanged following treatment with abiraterone acetate or CB 7627, but were markedly increased following ketoconazole, indicating no inhibition of corticosterone production by these steroidal compounds. ${ }^{[23]}$ 


\subsection{Therapeutic Trials}

\subsubsection{Cancer}

\section{Prostate Cancer}

Phase III: Abiraterone acetate increased survival by $36 \%$, compared with placebo, according to the results of a prespecified interim analysis of the COU-AA-301 trial. The trial included 1195 patients with metastatic, CRPC who had previously received one or two chemotherapy regimens, at least one of which contained docetaxel. Patients were randomized to receive abiraterone acetate or placebo, in addition to low-dose prednisone or prednisolone. The median overall survival duration was 14.8 months in the abiraterone group versus 10.9 months in the placebo group. The risk of death was reduced by $35 \%$ ( $p<0.001$ versus placebo). Abiraterone acetate also significantly increased the time to disease progression and PSA-response rate compared with placebo. ${ }^{[3]}$

Phase II : Radiologic disease control was achieved in 28 of $33(85 \%)$ evaluable patients, with partial responses in nine patients $(27 \%)$ and stable disease in 19 patients (58\%), according to preliminary results of a phase II trial (COU-AA-002) of abiraterone acetate plus prednisone in chemotherapy-naive patients with CRPC who had progressive disease despite treatment with $\mathrm{LHRH}$ analogs and other hormonal therapies. Patients received abiraterone acetate and prednisone once daily. After 12 weeks, a decline in levels of PSA of $>30 \%$ was noted in 26 patients $(79 \%$ ), a PSA decline of $>50 \%$ in 24 patients $(73 \%)$, and a PSA decline of $>90 \%$ in ten patients $(30 \%)$. The median time to PSA progression was 337 days. ${ }^{[1,12,24]}$

Preliminary results from a phase II trial (COU-AA-BMA) in patients with CRPC who had progressive disease despite treatment with LHRH analogs and other therapies showed that four patients (19\%) treated with abiraterone acetate in combination with prednisone showed an improvement in their bone scan after 6 months of treatment. A stable bone scan was seen in 15 patients $(71 \%) .{ }^{[24]}$

Preliminary results from a phase II trial (COU-AA-004) of abiraterone acetate in patients with metastatic CRPC have been reported. Abiraterone acetate in combination with prednisone was administered orally, once daily, to patients with CRPC who had progressive disease despite prior treatment with androgen deprivation therapy and first-line docetaxel-based chemotherapy. Of the 58 evaluable patients who have been treated so far, abiraterone acetate was well tolerated with only minimal toxicity. After 12 weeks, 20 patients (34\%) experienced a confirmed decline in PSA levels of $>50 \%$. Of the 31 patients who had not received prior treatment with ketoconazole, 13 patients $(42 \%)$ experienced a confirmed decline in PSA levels of $>50 \%$. Furthermore, of the 27 patients who had been previously treated with ketoconazole, seven patients $(26 \%)$ experienced a confirmed decline in PSA levels of $>50 \%$. The median time to PSA progression was estimated to be 198 days and 99 days in patients who had not been previously treated with ketoconazole and in those who had, respectively. Of the 18 patients with measurable tumor lesions, 11 patients $(61 \%)$ showed stable disease and three patients $(17 \%)$ had confirmed partial radiologic responses (by RECIST criteria). ${ }^{[1,27,28]}$

In a phase I/II trial (COU-AA-001) of abiraterone acetate in patients with hormone-refractory, chemotherapy-naive prostate cancer, abiraterone acetate was administered orally, once daily, to patients who had progressive disease despite treatment with LHRH analogs and multiple other hormonal therapies. At the time of PSA progression on abiraterone acetate, dexamethasone was added to abiraterone acetate to see if it could overcome the resistance to abiraterone acetate. Of the 54 evaluable patients from the study, 38 patients $(70 \%)$ experienced a confirmed decline in PSA levels of greater than $50 \%$ while receiving abiraterone acetate as a monotherapy. For the 54 evaluable patients in the trial, the median time to PSA progression while the patients were receiving abiraterone acetate alone was estimated to be 231 days ( 33 weeks). Thus far in the trial, 30 of the 54 patients have been treated with dexamethasone in addition to abiraterone acetate, at the time of PSA progression. Nine $(30 \%)$ of these 30 patients have experienced a confirmed partial decline in PSA levels of greater than $50 \%$ when treated with the combination of abiraterone acetate and dexamethasone after PSA progression on abiraterone acetate alone. 
Table II. History

\begin{tabular}{lll}
\hline Event date & Update type & Comment \\
\hline 11 October 2010 & Scientific update & Efficacy and adverse events data from a phase III trial in prostate \\
& cancer presented in a late-breaking presentation at the 35th \\
& Congress of the European Society for Medical Oncology (ESMO- \\
$2010)^{[3]}$
\end{tabular}

$\begin{array}{ll}30 \text { June } 2010 & \text { Phase change } \\ 21 \text { May } 2010 & \text { Trial update }\end{array}$

1 November 2009

10 July 2009

1 June 2009

30 April 2009

28 February 2009

11 February 2009

21 November 2008

18 October 2008

23 July 2008

15 July 2008

15 July 2008

15 July 2008

3 June 2008

29 April 2008

16 February 2008

31 July 2007

30 June 2007

18 June 2007

9 May 2007

29 December 2006

Phase change

12 December 2006
Phase change

Scientific update

Phase change

Phase change

Company name changes

Scientific update

Trial update

Scientific update

Regulatory status

Phase change

Scientific update

Scientific update

Phase change

Phase change

Phase change

Scientific update

Phase change

Scientific update

Scientific update

Scientific update
Phase I clinical trials in prostate cancer in Japan (PO)

Cougar Biotechnology completes enrollment in a phase III trial in metastatic castration-resistant prostate cancer in North America, Europe, and Australia

Phase II clinical trials in prostate cancer in the US (PO)

Cougar Biotechnology has been acquired by Johnson \& Johnson

Preliminary efficacy data from three phase II trials in prostate cancer presented at the 45th Annual Meeting of the American Society of Clinical Oncology (ASCO-2009) ${ }^{[1]}$

Cougar Biotechnology initiates a phase III trial in chemotherapynaive castration-resistant prostate cancer in North America, Europe, and Australia ${ }^{[8]}$

Interim efficacy and pharmacodynamics data from two phase II trials in prostate cancer released by Cougar Biotechnology[24]

Cougar Biotechnology and US FDA agree on Special Protocol Assessment for phase III trial of abiraterone acetate in castrationresistant, chemotherapy-naive prostate cancer ${ }^{[9]}$

Phase I/II clinical trials in advanced breast cancer in the UK (PO) Interim efficacy and pharmacodynamics data from a phase II trial in prostate cancer released by Cougar Biotechnology[12]

Efficacy data from a phase I trial in prostate cancer released by BTG plc. ${ }^{[17]}$

Phase III clinical trials in prostate cancer in Australia (PO)

Phase III clinical trials in prostate cancer in Canada (PO)

Phase III clinical trials in prostate cancer in Europe (PO)

Efficacy data from a phase I and phase II trials and pharmacokinetics data from a phase I trial in prostate cancer presented at the 44th Annual Meeting of the American Society of Clinical Oncology (ASCO-2008) ${ }^{[20,27,28]}$

Phase III clinical trials in prostate cancer in the US (PO)

29 April 2008

Efficacy data from a phase I trial in castration resistant prostate cancer presented at the 2008 ASCO Genitourinary Cancers Symposium ${ }^{[10]}$

Phase I clinical trials in prostate cancer in the US (PO)

Phase II clinical trials in prostate cancer in the US (PO)

Data presented at the 43rd Annual Meeting of the American Society of Clinical Oncology (ASCO-2007) added to the adverse events and cancer therapeutic trials sections ${ }^{[14]}$

Data presented at the 98th Annual Meeting of the American Association for Cancer Research (AACR-2007) added to the Cancer therapeutic trials section[29,30]

Phase II clinical trials in prostate cancer in the UK (PO)

5 October 2010

4 June 2010

26 March 2010

13 July 2009

11 June 2009

5 May 2009

3 March 2009

12 February 2009

24 November 2008

22 October 2008

23 July 2008

15 July 2008

15 July 2008

15 July 2008

13 June 2008

19 February 2008

9 November 2010 22 November 2007

29 June 2007

9 May 2007

29 December 2006

12 December 2006 prostate cancer have been added to the adverse events and cancer therapeutic trials sections ${ }^{[21]}$ 
Table II. Contd

\begin{tabular}{|c|c|c|c|}
\hline Event date & Update type & Comment & Update date \\
\hline 9 October 2006 & Scientific update & $\begin{array}{l}\text { Interim results from a phase I trial in patients with advanced } \\
\text { prostate cancer have been added to the adverse events and cancer } \\
\text { therapeutic trials sections }{ }^{[22]}\end{array}$ & 11 October 2006 \\
\hline 11 July 2006 & Phase change & Phase I clinical trials in prostate cancer in the US (PO) & 12 July 2006 \\
\hline 2 February 2006 & Regulatory status & $\begin{array}{l}\text { Cougar Biotechnology has filed an IND with the US FDA to conduct } \\
\text { a phase I trial for prostate cancer; the IND filing has been accepted }\end{array}$ & 7 February 2006 \\
\hline 14 December 2005 & Phase change & Phase $\mathrm{I} / \mathrm{Il}$ clinical trials for prostate cancer in the UK (PO) & 4 May 2006 \\
\hline 5 October 2005 & Regulatory status & $\begin{array}{l}\text { Cougar Biotechnology has filed a CTA with the MHRA to conduct a } \\
\text { phase I/II trial in the UK for prostate cancer; the CTA filing has been } \\
\text { accepted }\end{array}$ & 7 October 2005 \\
\hline 22 February 2005 & Scientific update & $\begin{array}{l}\text { Data from a media release have been added to the cancer } \\
\text { pharmacodynamics section }{ }^{[25]}\end{array}$ & 22 February 2005 \\
\hline 27 October 2004 & Scientific update & $\begin{array}{l}\text { Data from a media release have been added to the cancer } \\
\text { pharmacodynamics section }{ }^{[26]}\end{array}$ & 27 October 2004 \\
\hline 22 April 2004 & Licensing status & $\begin{array}{l}\text { Abiraterone acetate has been licensed to Cougar Biotechnologies } \\
\text { worldwide }\end{array}$ & 22 April 2004 \\
\hline 1 March 2001 & Licensing status & $\begin{array}{l}\text { Abiraterone acetate is available for licensing } \\
\text { (http://www.btgplc.com) }\end{array}$ & 1 March 2001 \\
\hline 18 December 1997 & Phase change & Phase I clinical trials for prostate cancer in the UK (PO) & 18 December 1997 \\
\hline 20 February 1996 & Phase change & Phase I clinical trials for cancer in the UK (PO) & 20 February 1996 \\
\hline 10 May 1995 & Phase change & Preclinical development for cancer in the UK (PO) & 10 May 1995 \\
\hline
\end{tabular}

The median time to PSA progression was estimated to be 399 days (57 weeks). Of the 29 evaluable patients with measureable tumor lesions, treatment with abiraterone acetate resulted in partial radiologic responses in 15 patients $(52 \%)$, with eight patients (28\%) demonstrating ongoing stable disease as assessed by an independent radiologic review blinded to outcome data. ${ }^{[27]}$

Radiologic disease control was achieved in 31 of $47(66 \%)$ evaluable patients with measurable tumor lesions, with partial radiologic response in six patients $(13 \%)$ and stable disease in 25 patients $(53 \%)$, according to results from a phase II trial (COU-AA-003) of once-daily abiraterone acetate in patients with CRPC who had progressive disease despite prior treatment with androgen deprivation therapy and first-line docetaxel-based chemotherapy. After 12 weeks, a decline in PSA levels of $>30 \%$ was observed in 24 patients $(51 \%)$, a PSA decline of $>50 \%$ in 19 patients $(40 \%)$, and a PSA decline of $>90 \%$ in six patients $(13 \%){ }^{[1,29]}$

Interim results indicated that abiraterone acetate (up to $2000 \mathrm{mg}$ /day, orally) provided clinical benefits in chemotherapy-naive patients with CRPC in a phase I/II study. Among 34 patients evaluable for response, 22 patients $(65 \%)$ had a confirmed decline in PSA levels of $>50 \%$, where 10 of 34 patients $(29 \%)$ had a decline in PSA levels of $>90 \%$. Among 20 evaluable patients with measurable tumor lesions, 11 patients $(55 \%)$ had partial radiologic responses, where seven patients showed durable stable disease and three patients had regression of bone lesions. Several abiraterone acetate recipients also had improvement in pain and a marked reduction in opiate use. Circulating tumor cells (CTC) were detected in 16 of 34 patients and changes in CTC counts were shown to correspond with changes in PSA. ${ }^{[14,30]}$

Phase I: During a phase I dose-ranging trial (COU-AA-002), abiraterone acetate was administered once-daily to chemotherapy-naive patients with CRPC who had progressive disease despite treatment with LHRH analogs and multiple other hormonal therapies. Overall, 27 of the 30 evaluable patients $(90 \%)$ experienced a decline in PSA levels while receiving abiraterone acetate, with 16 of 30 patients (53\%) experiencing a greater than $50 \%$ decline in PSA levels. Of the 11 patients in the trial who had not received prior ketoconazole treatment, six patients (55\%) experienced a greater 
Table III. Forecasts

\begin{tabular}{|c|c|c|c|}
\hline \multicolumn{4}{|c|}{ InThought Probability of Approval ${ }^{a}$} \\
\hline Indication & Approval Date Estimate & inThought Approvability Index & Last Update \\
\hline Prostate cancer & NE & $66 \%$ (NYR) & 21 Jul 2009 \\
\hline \multicolumn{4}{|c|}{$\begin{array}{l}\text { The Wolters Kluwer Health Approvability Index is a dynamic tool that assesses the progress of a drug candidate through clinical } \\
\text { development, evaluating strength of clinical data and trial design, benchmarked against historical parameters and likelihood to maintain } \\
\text { forward momentum. Points are assigned for specific line items relating to safety, efficacy, and other factors in each phase of clinical } \\
\text { development. Possible points total } 100 \text { upon drug approval, and are allocated in each phase according to the historical approval rate of } \\
\text { similar drugs, such that the current points of a drug relate to its probability of approval. In addition, a letter grade is assigned and reflects the } \\
\text { momentum of a drug candidate in its current phase, with 'A' indicating significantly above average/likely to progress, ' } C \text { ' indicating average, } \\
\text { and ' } F \text { ' indicating significantly below average/unlikely to progress. 'NYR' stands for 'Not Yet Rated,' indicating that the probability of } \\
\text { approval is based on historical approval rates for similar drugs according to indication, molecule type, novelty, and phase, but without } \\
\text { analyses of clinical data, trial design, and other factors specific to the individual agent. }\end{array}$} \\
\hline
\end{tabular}

$\mathrm{NE}=$ no estimate.

than $50 \%$ decline in PSA levels as a result of treatment with abiraterone acetate. Additionally, $10(53 \%)$ of the 19 patients who had previously received ketoconazole experienced a $50 \%$ or greater decline in PSA while receiving abiraterone acetate. Moreover, 9 (56\%) of 16 patients who had previously responded to ketoconazole also experienced a greater than $50 \%$ decline in PSA while being treated with abiraterone acetate and one $(33 \%)$ of the three patients who had no response to ketoconazole experienced a greater than $50 \%$ decline in PSA while being treated with abiraterone acetate. The median time to progression in the patients who had previously received ketoconazole was 21 weeks. ${ }^{[10,27]}$

Once-daily dosing of oral abiraterone acetate displayed clinical benefits as a second-line hormonal therapy in patients with CRPC in a phase I study $(n=18)$. Of the 13 patients that were evaluable for antitumor activity, 9 of $13(69 \%)$ and 6 of $13(46 \%)$ patients had confirmed reductions in PSA levels of $>50 \%$ and $>90 \%$, respectively. CTC were detected in 6 of 14 patients and changes in CTC counts correlated with changes in PSA. Among eight evaluable patients with measurable tumor lesions, abiraterone acetate therapy yielded partial radiologic responses in 5 of $8(63 \%)$ patients. In addition, individual patients treated with abiraterone acetate experienced radiographic regression of bone metastases and improvement in pain. ${ }^{[21,22]}$

Abiraterone acetate significantly reduced tumor volume and reduced PSA levels in $70-80 \%$ of chemotherapy-naive men with advanced prostate cancer resistant to multiple hormone therapies. ${ }^{[17]}$

\section{References}

1. Cougar Biotechnology Inc. Cougar Biotechnology Presents Positive CB7630 (Abiraterone Acetate) Phase II Data at ASCO Annual Meeting. www.cougarbiotechnology.com, 01 Jun 2009 Media Release

2. Johnson \& Johnson. Johnson \& Johnson Completes Acquisition of Cougar Biotechnology. www.jnj.com, $10 \mathrm{Jul}$ 2009 Media Release

3. Ortho Biotech Oncology Research \& Development. Abiraterone Acetate Significantly Improved Overall Survival for Patients with Metastatic Advanced Prostate Cancer. www.centocororthobiotech.com, 11 Oct 2010 Media Release

4. BTG, Cougar Biotechnology. BTG Licenses New Prostate Cancer Drug to Cougar Biotechnology. www.btgplc.com, 20 Apr 2004 Media Release

5. Cougar Biotechnology Inc. Cougar Biotechnology Announces Initiation of Phase III Trial of CB7630 (Abiraterone Acetate). www.cougarbiotechnology.com, $30 \mathrm{Apr}$ 2008 Media Release

6. BTG PLC. BTG plc: Abiraterone Acetate Meets PreDetermined Criteria in Metastatic Prostate Cancer Study. www.btgplc.com, 10 Sep 2010 Media Release

7. Ortho Biotech Oncology Research \& Development. Study of Investigational Agent Abiraterone Acetate for Metastatic Advanced Prostate Cancer Unblinded After Meeting PreDetermined Criteria. www.jnjpharmarnd.com, 09 Sep 2010 Media Release

8. Cougar Biotechnology Inc. Cougar Biotechnology Initiates Phase III Trial of CB7630 (Abiraterone Acetate) in Chemotherapy-Naïve Castration-Resistant Prostate Cancer Patients. www.cougarbiotechnology.com, 30 Apr $2009 \mathrm{Me}-$ dia Release

9. Cougar Biotechnology Inc. Cougar Biotechnology Announces Agreement with FDA on Special Protocol Assessment for Phase III Trial of CB7630 (Abiraterone Acetate) in Chemotherapy Naïve Castration Resistant Prostate Cancer Patients. www.cougarbiotechnology.com, 11 Feb 2009 Media Release

10. Cougar Biotechnology Inc. Cougar Biotechnology Announces Presentation of Positive CB7630 Phase I Clinical Data at ASCO 2008 Genitourinary Cancers Symposium. www.cougarbiotechnology.com, 16 Feb 2008 Media Release

11. Cougar Biotechnology Inc. Cougar Biotechnology Announces Initiation of Phase I Trial for CB7630 (Abiraterone Acetate). www.cougarbiotechnology.com, 11 Jul 2006 Media Release 
12. Cougar Biotechnology Inc. Positive Phase II Data on Cougar Biotechnology's CB7630 Presented at Prostate Cancer Foundation Scientific Retreat. www.cougarbiotechnology. com, 18 Oct 2008 Media Release

13. Cougar Biotechnology Inc. Cougar Biotechnology Presents Positive CB7630 Clinical Data at AACR Annual Meeting Late-Breaking Clinical Trials Session. www.cougarbio technology.com, 18 Apr 2007 Media Release

14. Cougar Biotechnology Inc. Cougar Biotechnology Announces Presentation of Positive CB7630 Clinical Data at ASCO Annual Meeting. www.cougarbiotechnology.com, 04 Jun 2007 Media Release

15. Cougar Biotechnology Inc. Cougar Biotechnology Initiates Phase II Trial of CB7630 (Abiraterone Acetate). www. cougarbiotechnology.com, 15 Dec 2006 Media Release

16. Phase 1 Study of JNJ-212082 (Abiraterone Acetate) in Patients With Castration-Resistant Prostate Cancer. Clinical Trial Profile

17. BTG PLC. BTG plc: Study Shows Abiraterone Can Successfully Treat Aggressive, Chemotherapy-Resistant Prostate Cancer. www.btgplc.com, 22 Jul 2008 Media Release

18. Cougar Biotechnology Inc. Cougar Biotechnology Reports 2008 Fourth Quarter and Year-End Financial Results. www.cougarbiotechnology.com, 17 Mar 2009 Media Release

19. Cougar Biotechnology Inc. Cougar Biotechnology Announces Initiation of Phase I/II Trial of CB7630 (Abiraterone Acetate) in Advanced Breast Cancer Patients. www.cougarbiotechnology.com, 21 Nov 2008 Media Release

20. Carden CP, Raynaud FI, Jones RL, et al. Crossover pharmacokinetics study to assess oral administration of abiraterone acetate capsule and tablet formulations in fasted and fed states in patients with prostate cancer. 44th Annual Meeting of the American Society of Clinical Oncology: (plus poster) abstr. 5168,30 May 2008. Available from URL: http://www.asco.org [English]

21. Cougar Biotechnology Inc. Cougar Biotechnology Presents Positive CB7630 Phase I data at the AACR Innovations in Prostate Cancer Research Conference. www.cougarbio technology.com, 09 Dec 2006 Media Release

22. Cougar Biotechnology Inc. Cougar Biotechnology Announces Positive CB7630 Phase I data Presented at the
National Cancer Research Institute Conference. $w w w$. cougarbiotechnology.com, 09 Oct 2006 Media Release

23. Barrie SE, Potter GA, Goddard PM, et al. Pharmacology of novel steroidal inhibitors of cytochrome P450(17 alpha) (17 alpha-hydroxylase/C17-20 lyase). J Steroid Biochem Mol Biol 1994; 50: 267-73

24. Cougar Biotechnology Inc. Cougar Biotechnology Announces Presentation of Positive CB7630 (Abiraterone Acetate) Phase II Data at ASCO 2009 Genitourinary Cancers Symposium. www.cougarbiotechnology.com, 28 Feb 2009 Media Release

25. Cougar Biotechnology Inc. Cougar Biotechnology Presents CB7630 Phase I Clinical Data at the 2005 Prostate Cancer Symposium. www.cougarbiotechnology.com, 21 Feb 2005 Media Release

26. Cougar Biotechnology Inc. Cougar Biotechnology Presents CB7630 Phase I Data at Prostate Cancer Foundation Scientific Retreat. www.cougarbiotechnology.com, $25 \mathrm{Oct}$ 2004 Media Release

27. Cougar Biotechnology Inc. Cougar Biotechnology Presents Positive CB7630 (Abiraterone Acetate) Phase I and Phase II Data at ASCO 2008 Annual Meeting. www.cougarbio technology.com, 03 Jun 2008 Media Release

28. Danila DC, Rathkopf DE, Morris MJ, et al. Abiraterone acetate and prednisone in patients with progressive metastatic castration resistant prostate cancer after failure of docetaxel-based chemotherapy. 44th Annual Meeting of the American Society of Clinical Oncology: (plus oral presentation) abstr. 5019, 30 May 2008. Available from URL: http://www.asco.org [English]

29. Reid AHM, Attard G, Molife R, et al. Abiraterone, an oral, irreversible CYP450c17 enzyme inhibitor appears to have activity in post-docetaxel castration refractory prostate cancer patients. 98th Annual Meeting of the American Association for Cancer Research: Clinical Research Track. (Spec. issue 3): abstr. LB-181, 14 Apr 2007. England [English]

30. Attard G, Reid AHM, Barrett M, et al. Inhibition of androgen synthesis results in a high response rate in castration refractory prostate cancer. 98th Annual Meeting of the American Association for Cancer Research: Clinical Research Track. (Spec. issue 3): abstr. LB-180, 14 Apr 2007. England [English] 